\title{
Modeling an unmitigated thermal quench event in a large field magnet in a DEMO Reactor
}

\author{
Brad J. Merrill ${ }^{*}$ \\ *Idaho National Laboratory, Idaho Falls, Idaho \\ P.O. Box 1625 \\ Idaho National Laboratory, Idaho Falls, Idaho, 83415, USA \\ Tel.: +12085260395 \\ E-mail address: Brad.Merrill@inl.gov (B.J. Merrill)
}

\begin{abstract}
The superconducting magnet systems of future fusion reactors, such as a Demonstration Power Plant (DEMO), will produce magnetic field energies in the 10s of GJ range. The release of this energy during a fault condition could produce arcs that can damage the magnets of these systems. The public safety consequences of such events must be explored for a DEMO reactor because the magnets are located near the DEMO's primary radioactive confinement barrier, the reactor's vacuum vessel (VV). Great care will be taken in the design of DEMO's magnet systems to detect and provide a rapid field energy dump to avoid any accidents conditions. During an event when a fault condition proceeds undetected, the potential of producing melting of the magnet exists. If molten material from the magnet impinges on the walls of the $\mathrm{VV}$, these walls could fail, resulting in a pathway for release of radioactive material from the VV. A model is under development at Idaho National Laboratory (INL) called MAGARC to investigate the consequences of this accident in a large toroidal field (TF) coil. Recent improvements to this model are described in this paper, along with predictions for a DEMO relevant event in a toroidal field magnet.
\end{abstract}

Keywords: fusion safety, unmitigated quench, magnet accident.

\section{Introduction}

The superconducting magnet systems of future fusion reactors, such as a DEMO, will produce magnetic field energies in the $10 \mathrm{~s}$ of GJ range. The release of this energy during a fault condition can produce arcs that can damage the magnets of these systems. A recent example of such events occurred in CERN's Large Hadron Collider (LHC) [1]. The public safety consequences of these events must be explored for a DEMO reactor because the magnets are located near the DEMO's primary radioactive confinement barrier, the reactor's VV.

Great care will be taken in the design of DEMO's magnet systems to detect and provide a rapid field energy dump to avoid accidents like the one that occurred at LHC. However, given recent events in the nuclear power industry, even beyond extremely unlikely events (frequency $<1 \times 10^{-6} / \mathrm{yr}$ ) must be considered for DEMO safety assessments. One such accident is an unmitigated quench event in a TF coil. During this event's progression, the fault condition proceeds undetected, with the potential of producing melting of the magnet. If molten material from the magnet impinges on the walls of the VV, these walls could fail, resulting in a pathway for release of radioactive material from the VV.

A model is under development by the Fusion Safety Program (FSP) at INL called MAGARC to investigate the consequences of this accident in a large TF coil [2,3]. Recent improvements to this model include the solution of Maxwell's Equations to predict the magnitude of induced currents produced in the magnet as the magnetic field decays. This model is described in this paper along with predictions for a DEMO relevant unmitigated quench event in a TF magnet.

In the following sections of this paper, a description of an unmitigated quench event will be given, the modeling approach and equations solved by MAGARC will be discussed, and the predicted results of applying the most recent version of MAGARC to an unmitigated quench in a large TF coil will be presented. The final section summarizes the findings.

\section{Superconducting toroidal field coils and an unmitigated an quench event}

As described in [2], the superconducting TF coils of a large tokamak reactor operate at a maximum field in excess of $12 \mathrm{~T}$, a maximum current of $70 \mathrm{kA}$, and a temperature of $4.5 \mathrm{~K}$. For a set of $20 \mathrm{TF}$ coil set, the total stored magnetic field energy is in excess of $100 \mathrm{GJ}$.

Figure 1 contains a cross-sectional view of a large TF coil design as modelled by MAGARC. Shown in this figure are the coil case and a winding pack composed of seven radial steel plates with machined grooves supporting circular magnet conductor in a pancake configuration. The magnet's insulation provides electrical isolation between the superconducting cables and the radial plate of a pancake, between adjacent pancakes, and between the winding pack pancakes and the coil case.

*Brad.Merrill@inl.gov 
In this example, the superconducting cables are composed of $\mathrm{Nb}_{3} \mathrm{Sn}$ superconductor and copper stabilizing strands twisted together, with the copper strands providing quench protection. These cables are wrapped around a perforated steel helium cooling tube that allows the helium to flow radially through the current carrying superconductor and copper strands. The cables and helium cooling tube are encased in an Incoloy jacket. The cables of the first and last turns of each pancake transition through the outer edge of the outboard coil case where they are joined to adjacent pancakes. The first turn of the first pancake and the last turn of the final pancake are connected to the magnet's current leads (busbars).

A TF magnet quench can occur when the magnet's superconductor changes from the superconducting to the normally conducting state. In the superconducting state the electrical resistivity of the conductor drops to near zero. This state occurs within a limited domain characterized by a critical temperature, a critical magnetic field, and a critical current density. If an event causes temperature or current density to exceed the boundaries of the superconducting region, the superconductor returns to a normal state with a finite resistivity. This transition could be produced by frictional heating during normal operation. During this type of transition, the quench is stabilized by the cables' copper strands, which have an electrical resistivity that is less than the superconductor in the normal state with the capacity to carry the entire coil current until the superconductor temperature drops to superconducting levels. More dramatic failures that cause thermal quenches are electrical shorts from insulation cracking, magnet power source faults, or as was the case for LHC, the failure of a joint in the superconducting cable. These quenches can be mitigated by an active system that rapidly discharges the coil set current through a large bank of external resistors.

An unmitigated TF magnet quench occurs when the quench continues undetected due to postulated safety detection and mitigation system failures. This is clearly a beyond extremely unlikely event because it involves safety system failures. However, if such an event were to occur, the quenched conductor location would experience resistive heating and undergo a temperature rise that would result in melting of the copper stabilizer within 20 to $30 \mathrm{~s}$. After the copper stabilizer melts and begins to vaporize, the coil current would be forced to arc between the ends of the intact conductor on either side of the melt zone. This "in-line" arc would produce $\sim 20 \mathrm{MW}$ of local heating based on experimental data [4], and cause adjacent magnet turns to quench. As the accident progresses, the in-line arc would grow in length. Eventually two bypass arcs with a lower-voltage total drop would form between the arcing conductor ends and radial plate, thus including the plate as part of the arc/current path [5].

Assuming that the cable's outer insulation wrap is still intact, the shortest average distance for a bypass arc to traverse, from the cable to a magnet's radial plate, is that of the radius of the cable, which in the case of this magnet design is $20 \mathrm{~mm}$. Because the resistance to current flow in the large radial plate is much lower than that of an arc, once an in-line arc reaches a length of 40 $\mathrm{mm}$ in this magnet design case, the arc current should break into two bypass arcs in order to follow a path of least resistance. Of course this is a very simplistic modeling assumption that requires additional improvement in a future version of this code.

As the temperature of the radial plate increases due to the ohmic heating caused by the bypass current and the bypass arcs, the insulation in the gap between this radial plate and the adjacent plate, which is $4 \mathrm{~mm}$ wide in this example, will eventually fail and arcs in this gap will also form once bypass arcs form in the windings of the adjacent plate. These in-line, bypass and gap arcs will continue, driven by the 100 GJ of inductive magnetic energy stored in the TF magnet set, until the magnetic energy is resistively dissipated in the quenching coil or the arc leaves the magnets by way of the magnet leads. The safety concern of this event is not magnet damage by melting, but rather the possibility that this molten material could be ejected from the magnet and impinge on the $\mathrm{VV}$, thereby challenging the integrity of this vessel.

\section{Equations solved by MAGARC to model an unmitigated magnet quench}

As detailed in [2], MAGARC solves a threedimensional (3-D) heat conduction equation in Cartesian coordinates to simulate the thermal response of the radial plates, coil case, and associated magnet insulation. Coupled with this three-dimensional radial plate thermal model is the solution of a quasi-two-dimensional (1-1/2 D, lumped in the radial direction) cylindrical heat conduction equation that represents the magnet's entire length of superconducting cable. This approach was taken because of the partially decoupled thermal response of the superconducting cables and radial plate of a coil pancake. This decoupling is associated with the electrical insulation wrapped around the outside of the cable jacket, which also acts as a good thermal insulator. This is a crucial modeling issue because the quench front primarily propagates along the axis (axial direction) of these windings. The $3-\mathrm{D}$ heat conduction mesh of the MAGARC code is nodalized such that each temperature cell of the magnet's windings, represented by the $1-1 / 2$ D conduction equation, fits within a corresponding computational cell in the $3-\mathrm{D}$ mesh in the axial direction.

In the initial version of MAGARC, the arcing behavior was simulated by a 3-D resistive circuit model developed for the radial plates and coil case based on the node voltage method [2]. The resistive circuit network for this model spans the same locations as the 3-D thermal heat conduction model presented above. Recently, the solution of the vector potential form of Maxwell's Equations [6] was added to this same 3-D mesh, with the goal of creating a more realistic simulation of the voltages and currents that developed arcs during an unmitigated coil quench event [3]. The 
vector potential equation relating magnetic vector and electric scalar potentials can be written as follows:

$$
\nabla \times \frac{1}{\mu} \nabla \times \overrightarrow{\mathrm{A}}=-\sigma\left(\nabla \phi+\frac{\partial \overrightarrow{\mathrm{A}}}{\partial \mathrm{t}}\right)+\overrightarrow{\mathrm{j}}_{\mathrm{c}}
$$

where $\mu$ is the material magnetic permeability $(H / m), \vec{A}$ is the magnetic vector potential $(\mathrm{Wb} / \mathrm{m}), \sigma$ is the material electrical conductivity $\left(\mathrm{ohm}-\mathrm{m}^{-1}\right), \phi$ is the electric scalar potential (V) and $\vec{j}_{\mathrm{c}}$ is a current density source $\left(\mathrm{A} / \mathrm{m}^{2}\right)$ supplied by an external power supply that is independent of the fields being calculated within the magnet, such as the current flowing in the superconductor of the magnet.

Equation 1 can be written in a different form, by making use of the vector identity $\nabla \times \nabla \times \overrightarrow{\mathrm{A}}=-\nabla^{2} \overrightarrow{\mathrm{A}}+\nabla(\nabla \cdot \overrightarrow{\mathrm{A}})$ and applying the Coulomb gage condition of $\nabla \cdot \overrightarrow{\mathrm{A}}=0$, as follows:

$$
\sigma \frac{\partial \overrightarrow{\mathrm{A}}}{\partial \mathrm{t}}=\frac{1}{\mu} \nabla^{2} \overrightarrow{\mathrm{A}}-\sigma \nabla \phi+\overrightarrow{\mathrm{j}}_{\mathrm{c}}
$$

Equation 2 is solved for the magnetic vector potentials on the $3-\mathrm{D}$ heat conduction equation mesh with the components of the vector potential and currents defined at the cell faces, and the electrical scalar potential defined at the cell center (e.g., at the same location as the temperature). Equation 2 must be solved under the following constraint that current is conserved:

$$
\begin{aligned}
\nabla \cdot \overrightarrow{\mathrm{j}} & =\nabla \cdot \overrightarrow{\mathrm{j}}_{\mathrm{o}}+\nabla \cdot \overrightarrow{\mathrm{j}}_{\mathrm{c}} \\
& =-\nabla \cdot \sigma \nabla \phi-\nabla \cdot \sigma \frac{\partial \vec{A}}{\partial t}+\nabla \cdot \overrightarrow{\mathrm{j}}_{\mathrm{c}}=0
\end{aligned}
$$

Here, the solution of Equation 3 gives the electric scalar potential.

Once an arc initiates in the MAGARC, the arc voltage drop is defined as a function of gap width and current density based on the following correlation of data obtained for confined arcs (e.g. arcs that are enclosed by structure) in a superconducting cable [4]:

$$
V_{\text {arc }}=V_{o}+\Delta_{\text {arc }}\left(1.75+0.012 \mathrm{j}_{\mathrm{arc}}^{1.75}\right)
$$

where $V_{\text {arc }}$ is the arc voltage drop $(\mathrm{V}), V_{o}$ is the minimum voltage required to initiate an arc (V), $\Delta_{\text {arc }}$ is the length of the $\operatorname{arc}(\mathrm{mm})$ and $\mathrm{j}_{\mathrm{arc}}$ is the arc current density $\left(\mathrm{A} / \mathrm{mm}^{2}\right)$.

The area of an arc $\left(\mathrm{A}_{\text {arc }}, \mathrm{mm}^{2}\right)$ is obtained from a simple fit to data found in [4], which is:

$$
A_{\text {arc }}=63.8+0.07 I_{\text {arc }}
$$

where the $I_{\text {arc }}$ is the current flowing in the intact cable at the location of the arc, with the constraint that the arc area is not allowed to exceed the cross-sectional area of the cable for in-line and bypass arcs. For the example configuration of Figure 1, the length of a bypass arc is 20 $\mathrm{mm}$. Therefore the minimum length of an in-line arc is $40 \mathrm{~mm}$. The length of a gap arc is $4 \mathrm{~mm}$. Equations 4 and 5 are used to determine the arc electrical conductivity used in the solution of Equation 3 at a cell face where an arc forms during an unmitigated quench.

After the insulation in a gap, either between plates or between the plates and coil case, fails, which for MAGARC is at a temperature of $900{ }^{\circ} \mathrm{C}$ [3], arcs are allowed to form at that cell face location. Because multiple insulation failures can occur in the same gap, MAGARC must employ a method to limit the number of arcs in that gap to a value that can be physically sustained. The empirically derived criterion adopted to do this is known as the 'Steenbeck minimum principle' which states that the electric field strength along an arc column assumes a minimum value for a given current and other conditions necessary to maintain the arc discharge. Based on data from external or unconfined arcs, the adopted minimum electric field occurs at a current of $\sim 3 \mathrm{kA}$ [3]. This means that if $30 \mathrm{kA}$ of current was predicted to arc across a common gap that a maximum of 10 arcs would be allowed to form in this gap. If the current drops even slightly below $30 \mathrm{kA}$ but above $27 \mathrm{kA}$, the number would be 9 arcs. An $11^{\text {th }}$ arc would not form until the current reaches $33 \mathrm{kA}$.

\section{The application of MAGARC for an unmitigated magnet quench}

Figure 2 contains results of a comparison between MAGARC predictions for an unmitigated-quench scenario in a large TF coil with and without the new electromagnetics capability invoked. The consistent factor that explains the difference in this comparison is the existence of induced eddy currents for the case with the electromagnetics model.

These induced currents result in structural heating in coil locations where heat is not predicted for the case without the electromagnetics model. This additional heating causes superconducting coils to quench sooner in these coil locations. This can be clearly seen in Figure $2 \mathrm{c}$ which shows a complete quench of the TF coil at $\sim 40 \mathrm{~s}$, which is $60 \mathrm{~s}$ sooner than the case without the electromagnetics model invoked.

The more rapid quench for the electromagnetics model case produced a larger voltage drop across the leads of the magnet $(15 \mathrm{kV}$ vs. $4.5 \mathrm{kV})$ as more of the magnet's superconducting coil became resistive at an earlier time in the transient. In addition, arcs form earlier (Figure 2b) and the magnet current exits the magnet sooner by way of an arc in the magnet's busbar (Figure 2a) in the case with the electromagnetics model. Since the magnet current leaves the magnet sooner, then the amount of energy deposited in the magnet is also less (12 GJ vs. 13.4 GJ, note Figure 2a).

Correspondingly, the magnet melt volume is also less $\left(0.08 \mathrm{~m}^{3}\right.$ vs. $0.28 \mathrm{~m}^{3}$ or $\sim 3$ times less melt (note Figure $2 \mathrm{~d}$ ) when induced currents are considered for two reasons: 1) because the energy deposition is slightly less $(\sim 10 \%)$, and more importantly 2$)$ because the energy deposition is more uniformly distributed throughout the entire magnet resulting in more uniform structural 
heating instead of producing localized heating and melting. However in either case, the predicted melt volumes should not be sufficient to cause VV structural damage in excess of that already demonstrated by previous safety analyses [2].
These new results are encouraging from the standpoint of reactor safety because previous MAGARC predictions [2] showed that nearly $83 \%$ of the TF magnet set stored magnetic energy would be deposited in the quenching

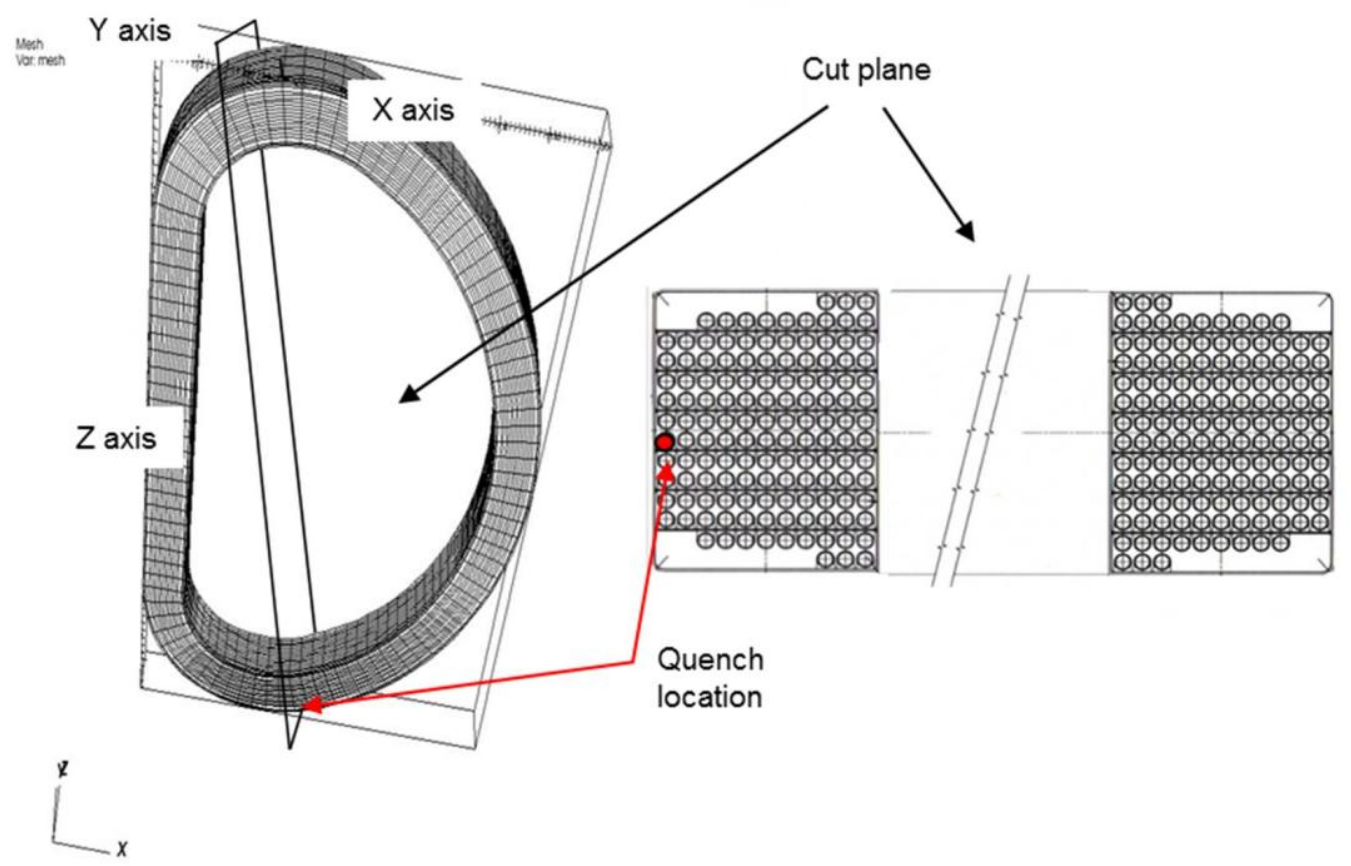

Fig. 1. Example of a MAGARC large TF magnet computational mesh showing the location of quench initiation (note: MAGARC mesh, which is rectangular, is mapped here onto a realistic magnet geometry for display purposes only). 


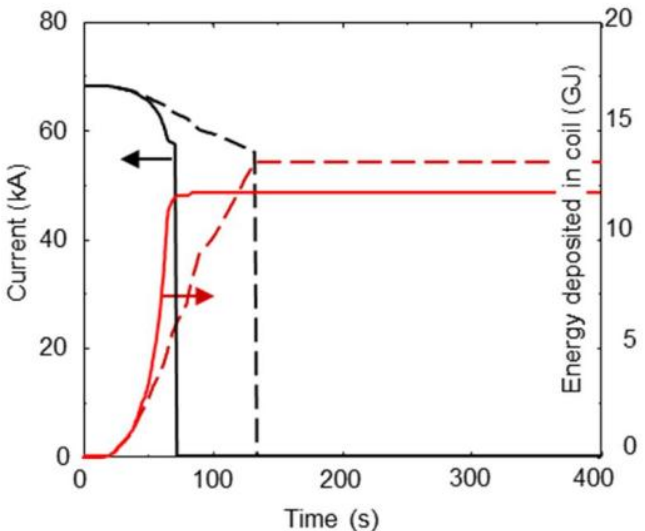

(a)

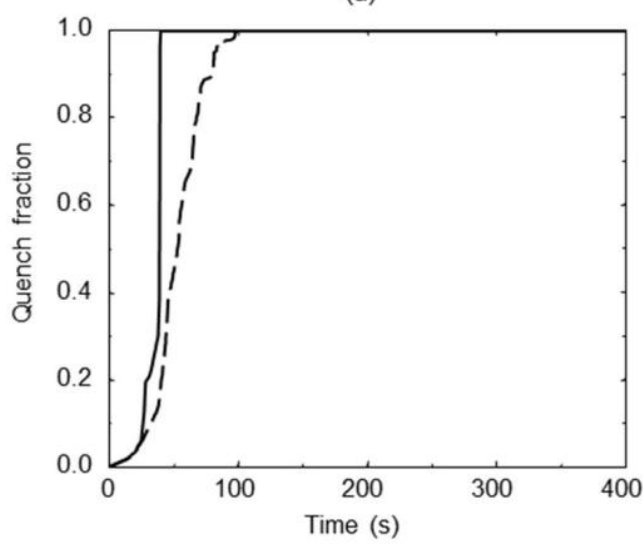

(c)

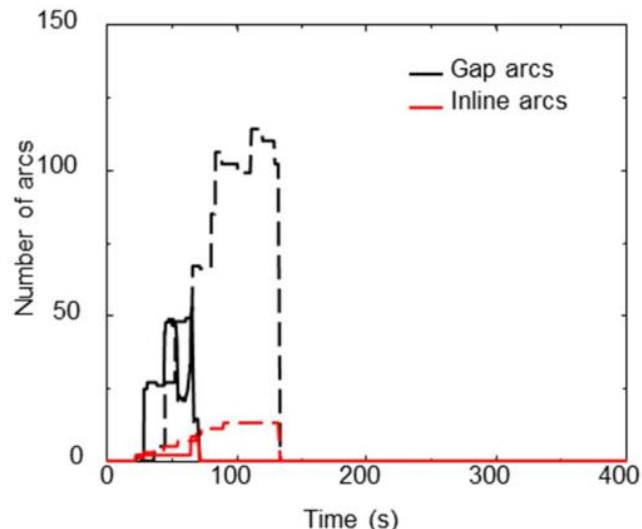

(b)

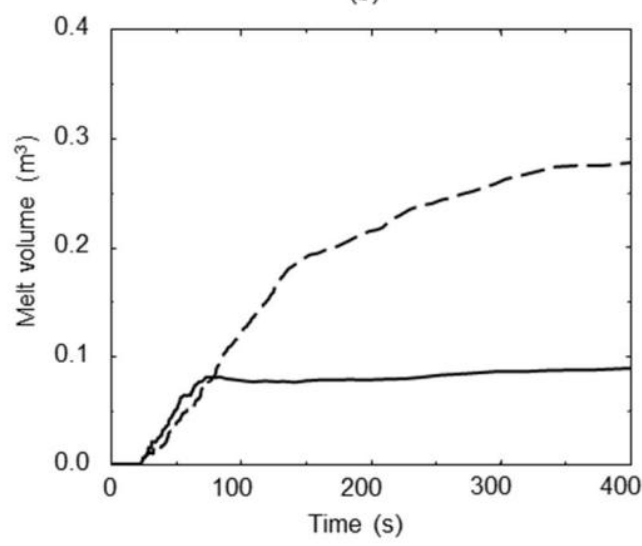

(d)

Fig. 2. MAGARC results for an unmitigated quench event comparing the predictions with induced currents (solid line) and without induced currents (dotted line) for: a) coil current, b) number of arcs, c) fraction of magnet quenched, and d) volume of melt, all versus time.

magnet as compared to $\sim 10-15 \%$ shown here. However, based on these results, the safety implications of an unmitigated TF magnet quench now shifts from magnet melt volume to the fact that $\sim 85 \%$ of the magnetic field energy is now available to cause damage to the busbars, cryostat, other TF coils, and TF power supplies.

\section{Summary}

A detailed model called MAGARC for predicting the consequences of an unmitigated quench in a large TF magnet is being developed by the FSP at the INL. MAGARC accounts for the phenomena of arcing, structure currents, melting, and thermal conduction. A recent version of this code has been developed with improved arcing logic and the ability to include eddy currents produced by the collapse of the magnetic field produced by during this event.

An unmitigated quench is a beyond extremely unlikely event that for a DEMO TF magnet is a significant accident because a large fraction of the 100 GJ of stored magnetic field energy of the TF coil set could be resistively dissipated in the quenched coil by the currents in the TF magnet system. As improvements to MACARC are made over time, the predicted fraction of the energy deposited in this magnet has decreased significantly from initial estimates of $\sim 83 \%$ to the recent predictions of this paper being as low as $\sim 10-15 \%$. This reduction in deposited energy produces a much lower magnet melt volume, which if it were to impinge on a DEMO VV would not likely fail this primary radioactive confinement boundary.

However, in the absence of test data from a superconducting magnet quench event to validate many of the details of MAGARC, additional peer review and benchmarking is needed. This is a primary purpose of publishing this paper at this time.

\section{Acknowledgments}

This material is based upon work supported by the $\mathrm{U}$. S. Department of Energy Office of Science, Office of Fusion Energy Sciences, under the DOE Idaho Operations Office contract number DE-AC07$05 I D 14517$.

\section{References}

[1] L. Rossi, Superconductivity: its role, its success and its 
setbacks in the Large Hadron Collider of CERN, Superconductor Science and Technology, 23 (2010).

[2] B. J. Merrill, Modeling an unmitigated quench event in an ITER toroidal field magnet, Fusion Technology, 37 (2000) 231-246.

[3] B. J. Merrill, Final report on ITER task agreement 81-10, Idaho National Laboratory Report, INL/EXT-09-15292. (2009).

[4] H. Kronhardt, Einfluß von Kurzschlüssen und Lichtbögen auf die Sicherheit von Magnetsystemen, Insitut für Technische Physik, Kfk 5096, S. 49, (1993).

[5] D. Klimenko, V. Pasler, Safety of fusion magnets: Model experiments to high current arcs at ITER busbars and feeder lines, Fusion Engineering and Design 87 (2012) 675-679.

[6] R. J. Thome, J. M. Tarrh, MHD and fusion magnets: Field and force design concepts, John Wiley \& Sons, Inc., New York, New York, (1982) 330-331. 


$$
\text { ON }
$$




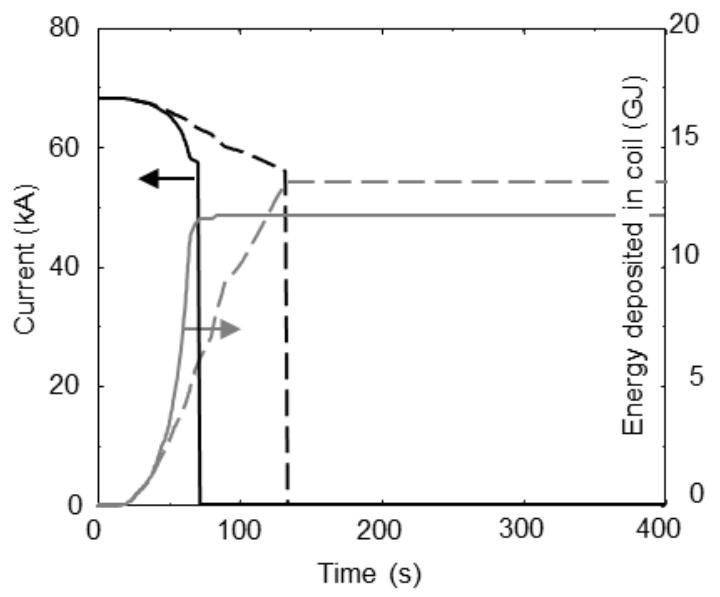

Fig. 2a 


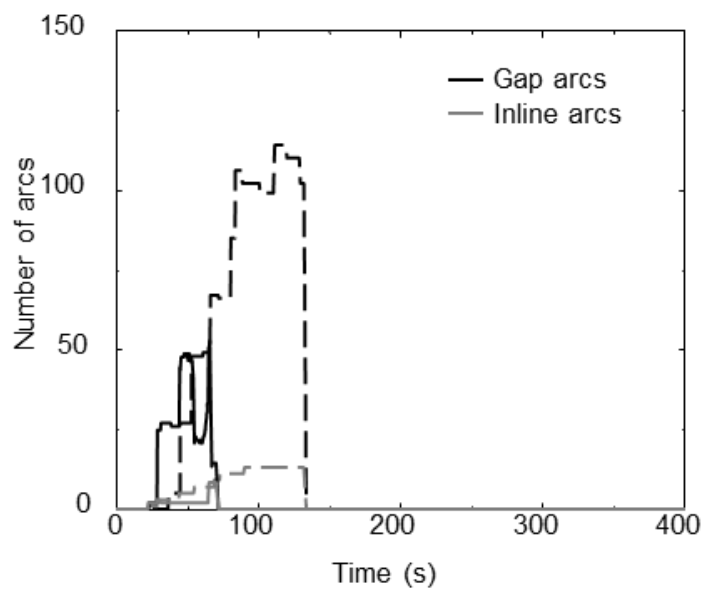

Fig. 2b 


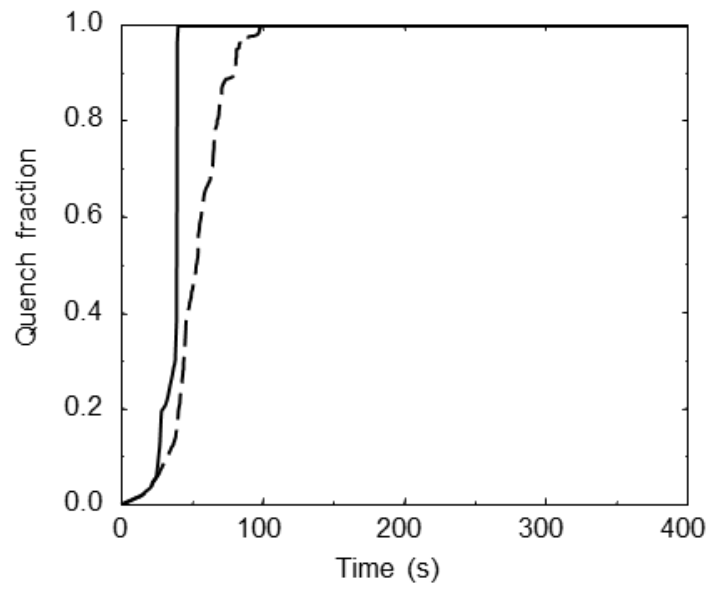

Fig. 2c 


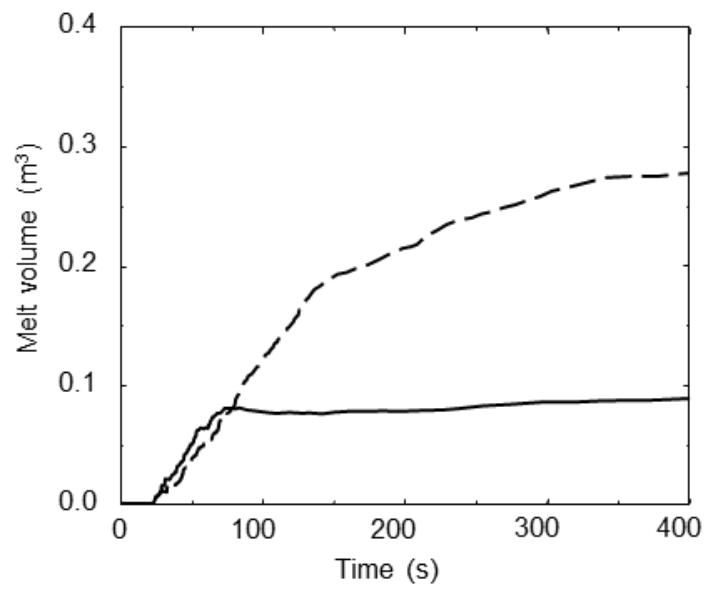

Fig. 2d 